\title{
TNFa and MMP1 in brain microvascular endothelial cells regulate blood-brain barrier dysfunction in psychotic disorders
}

Paulo Lizano ( $\square$ lizanopl@gmail.com )

BIDMC https://orcid.org/0000-0002-7128-6697

\section{Sovannarath Pong}

BIDMC

\section{Stephanie Santarriaga}

MGH

\section{Deepthi Bannai}

BIDMC

\section{Rakesh Karmacharya}

Harvard University https://orcid.org/0000-0002-0565-5482

\section{Article}

Keywords: Psychosis, Schizophrenia, Bipolar disorder, brain microvascular endothelial cells, blood brain barrier, MMP1, TNFa

Posted Date: February 14th, 2022

DOI: https://doi.org/10.21203/rs.3.rs-1162029/v1

License: (9) (1) This work is licensed under a Creative Commons Attribution 4.0 International License.

Read Full License 
Title: TNF $\alpha$ and MMP1 in brain microvascular endothelial cells regulate blood-brain barrier dysfunction in psychotic disorders

Authors: Paulo Lizano $0^{1,2,3,4^{+}, *}$, Sovannarath Pong ${ }^{1,2,3+}$, Stephanie Santarriaga ${ }^{3,4,5}$, Deepthi Bannai ${ }^{1,2}$, and Rakesh Karmacharya ${ }^{3,4,5,6 *}$

\section{+Co-First Authors}

\section{*Corresponding Authors:}

Paulo Lizano MD, PhD, 75 Fenwood Road, Room 612, Boston, MA 02115, Phone: 617-754-1227, Fax: 617-754-1250, lizanopl@gmail.com

Rakesh Karmacharya MD, PhD, 185 Cambridge Street, CPZN 6.226, Boston, MA 02114, Phone: 617726-5119, Fax: 617-726-0830, karmacharya@mgh.harvard.edu

${ }^{1}$ Department of Psychiatry, Beth Israel Deaconess Medical Center, Boston, MA, USA

${ }^{2}$ Division of Translational Neuroscience, Beth Israel Deaconess Medical Center, Boston, MA, USA

${ }^{3}$ Center for Genomic Medicine, Massachusetts General Hospital, Boston, MA, USA

${ }^{4}$ Department of Psychiatry, Harvard Medical School, Boston, MA, USA

${ }^{5}$ Chemical Biology and Therapeutic Science Program, Broad Institute of MIT and Harvard, Cambridge, MA, USA

${ }^{6}$ Schizophrenia and Bipolar Disorder Program, McLean Hospital, Belmont, MA, USA

Keywords: Psychosis, Schizophrenia, Bipolar disorder, brain microvascular endothelial cells, blood brain barrier, MMP1, TNF $\alpha$

\section{Abstract word count: 147}

Main text word count: 3991

Figures: 4

Tables: 0

Supp Figures:2

Supp Tables: 5 


\section{Abstract}

In schizophrenia and bipolar disorder, tight junction deficits in brain microvascular endothelial cells (BMECs) lead to blood brain barrier (BBB) dysfunction. Using an in vitro model of human BBB function, we show that $B M E C s$ derived from human induced pluripotent stem cells of schizophrenia patients show reduced $B B B$ integrity and increased small molecule permeability when compared to healthy control BMECs. Stratification based on BBB function in schizophrenia and bipolar disorder patients identified a BBB-deficit subtype with reduced barrier function, increased permeability to larger molecules, and decreased claudin-5 (CLDN5) levels. BMECs from the BBB-deficit group show increased MMP1 activity, which correlated with reduced CLDN5 levels and worse BBB function, which were rescued by tumor necrosis factor $\alpha$ (TNF $\alpha$ ) and MMP1 inhibition. These results show intrinsic deficits in BMECs in psychotic disorders that result in BBB disruption and further identify TNF $\alpha$ and MMP1 as potential targets for ameliorating BBB deficits.

\section{Introduction}

Schizophrenia (SZ) and bipolar disorder (BD) account for a large proportion of morbidity, mortality, and global burden associated with severe mental disorders ${ }^{1}$. Despite a significant genetic overlap between $\mathrm{SZ}$ and $\mathrm{BD}^{2}$, little clarity exists regarding their pathogenesis. Although there is convergent evidence for blood brain barrier (BBB) dysfunction and peripheral inflammation in $\mathrm{SZ}$ and $\mathrm{BD}$, it remains to be determined whether BBB deficits are 1) intrinsic to brain microvascular endothelial cells (BMECs) which results in increased peripheral inflammation or 2) whether peripheral inflammation from other sources impair BBB function ${ }^{3}$. The BBB protects the brain by regulating molecular and cellular trafficking between the bloodstream and brain parenchyma through physical and enzymatic transport, as well as by regulating immunological processes that are vital for brain homeostasis and response to injury ${ }^{3}$. Notably, the BBB is composed of a monolayer of BMECs that line blood vessels and provide strict regulation of paracellular permeability through protein junctional complexes between BMECs that limit passive diffusion and paracellular transport ${ }^{4}$.

Findings from peripheral inflammatory cytokine, transcriptomic, genetic, and neuroimaging studies point to an important role for BMECs in the pathogenesis of $S Z$ and $\mathrm{BD}^{3}$. For example, circulating peripheral inflammatory cytokines have been shown to negatively impact the BBB $^{5}$. Conversely, transcriptomic and genetic studies indicate the presence of disruptions in paracellular ${ }^{6}$ and transcellular $^{7-13}$ mechanisms related to BMEC function. There is also increasing evidence for increased $\mathrm{BBB}$ permeability in subgroups of patients with $\mathrm{SZ}^{7,14,15}$ and $\mathrm{BD}^{16,17}$. These findings suggest $\mathrm{BMEC}$ involvement in the pathogenesis of SZ and BD in specific subgroups of patients, but there is no study to date that has directly investigated the role of BMECs in SZ and BD. While, there is no consensus on which models are best for interrogating BBB function, stem cell-based BBB models are promising avenues since they are able to recapitulate the intrinsic contribution of disease- and patient-specific genetic backgrounds ${ }^{3,18}$. We recently adapted and validated a method to model BBB function in vitro using human induced pluripotent stem cell (iPSC)-derived BMECs ${ }^{19}$, in order to gain new insights into the biology underlying SZ and BD.

In this study, we employ human iPSC-derived BMECs to investigate BBB function in SZ and psychotic BD (collectively referred to as psychotic disorders). We report here the presence of increased BBB permeability in BMECs from SZ patients compared to healthy control BMECs and show that stratification based on BBB function can be used to identify a "BBB-deficit" subtype of psychosis patients that have $B M E C$ biology that is distinctly different from BBB-nondeficit patients or healthy individuals. This BBB- 
deficit group demonstrated increased MMP1 levels and activity, which was associated with impaired BBB function. Pharmacological inhibition of MMP1 with piroxicam or biological inhibition of TNF $\alpha$ with infliximab, an upstream regulator of MMP1, were able to restore BBB function in BBB-deficit patients.

\section{Results}

Morphological and functional properties in an in vitro human BBB model. The impervious nature of the human BBB is attributed primarily to the structure and function of BMECs, comprising physical, enzymatic, transport, and immunological processes that are vital for proper BBB functioning ${ }^{3}$. We had previously adapted and optimized a protocol for differentiating human iPSCs to generate BMEC-like cells with morphological and molecular marker expression (TJP1, OCLN, CLDN5, CD31 and SLC2A1), physical barrier properties with high transendothelial electrical resistance (TEER) $\geq 2000 \Omega \times \mathrm{cm}^{2}$, enzymatic transport activity for $A B C B 1$ and $A B C C 1$, and angiogenic potential in the sprouting assay that are characteristic features of BMECs ${ }^{19-21}$. Human iPSCs were grown in Essential 6 (E6) medium for four days to prime the cells for differentiation and then switched to human endothelial serum-free culture medium (hESFM) supplemented with $20 \mathrm{ng} / \mathrm{mL}$ basic fibroblast growth factor (bFGF), $10 \mu \mathrm{M}$ retinoic acid (RA), and B27 supplement to induce BMEC differentiation ${ }^{19}$. On the sixth day, cells were sub-cultured onto a collagen IV and fibronectin 1 matrix hESFM plus B27, bFGF, and RA resulting in a purified BMEC population by day $8^{21}$ (Fig. 1a).

We utilized a transwell system to examine the physiological properties of the BBB and quantify TEER, a standard measure of barrier properties (Fig. 1b). BMECs were differentiated from human iPSCs of four healthy control (HC), four psychotic bipolar I disorder (BD), and four schizophrenia (SZ) patients that were matched for age and sex (Supplementary Table 1). In order to establish the reliability of TEER measurements both within and across different cell lines, TEER experiments were conducted with technical as well as biological replicates over the course of six days. TEER was peaked on day 2 (TEER $\geq$ 2,000 $\Omega \times \mathrm{cm}^{2}$ ) and plateaued on day 3 (TEER $\sim 1,000 \Omega \times \mathrm{cm}^{2}$ ), as had been described previously for iPSC-derived BMECs ${ }^{22,23}$. Additionally, low standard errors $\left(10.4-339 \Omega \times \mathrm{cm}^{2}\right)$ were observed across technical and biological replicates, demonstrating consistent TEER function across HC, BD or SZ cell lines (Supplementary Fig. 1a). TEER experiments were performed for each of the 12 cell lines, with an average of three biological replicates and across six days. The results demonstrate similar individual variation in TEER function across all patients in the study with average standard errors of $45.8 \Omega \times \mathrm{cm}^{2}, 75.6 \Omega \times$ $\mathrm{cm}^{2}$, and $77.9 \Omega \times \mathrm{cm}^{2}$ for $\mathrm{HC}, \mathrm{BD}$, and SZ, respectively (Supplementary Fig. 1b). This demonstrates that BMECs across all groups have a maximal peak TEER on day 2 , followed by sustained TEER elevations over 6 days, as well as minimal variation within and between experimental conditions, supporting the use of TEER as a primary functional outcome for measuring BBB function.

BBB barrier dysfunction in SZ. We sought to determine whether BMECs could recapitulate BBB disruption described in human postmortem and in vivo studies ${ }^{7,16,24}$. We found robust evidence of BBB disruption in SZ and BD BMECs, with significant reduction in peak TEER values primarily when compared to HC BMECs (Fig. 1C). Studies examining BBB function in similar iPSC derived BMEC usually collect TEER values 2-3 days after subculturing2/11/2022 4:32:00 PM ${ }^{20,22,23}$. Thus, we compared TEER values on day 2 and found a significant reduction in the SZ BMECs compared to the HC BMECs. There was no difference identified between the BD group and SZ or HC groups (Fig. $1 \mathrm{~d}$ ). Since the BBB is also characterized by impermeability ${ }^{20,21}$, we performed a paracellular permeability assay to determine BBB leakiness using fluorescently labeled molecules ranging from $0.1 \mathrm{kDa}$ to $80 \mathrm{kDa}$ (Fig. 1e) ${ }^{25}$. SZ BMECs showed greater permeability to $0.1 \mathrm{kDa}$ and $10 \mathrm{kDa}$ molecules when compared to $\mathrm{HC}$ or BD BMECs (Fig. 1f). Interestingly, SZ BMECs showed higher permeability to $70 \mathrm{kDa}$ and $80 \mathrm{kDa}$ molecules compared to BD but not to HC 
BMECs (Fig. 1f). These results demonstrate that BMECs from SZ patients result in poorer BBB integrity as evidenced by lower TEER and higher permeability compared to HC or BD BMECs.

Paracellular transport is controlled by a complex arrangement of tight junction proteins in BMECs including CLDN5, OCLN, and TJP1 that are vital for maintaining BBB integrity ${ }^{3}$. Several studies have identified paracellular deficits in SZ, particularly as it relates to CLDN5 ${ }^{3}$. CLDN5 is deleted in 22q11 deletion syndrome (22qDS) which carries a $30 \%$ risk for developing $\mathrm{SZ}^{26}$ and $60 \%$ risk for developing $\mathrm{BD}^{27}$. Also, BMECs from patients with $22 \mathrm{qDS}+\mathrm{SZ}$ showed impaired BBB integrity ${ }^{23}$. In animal models CLDN5 knockdown results in increased BBB leakage ${ }^{6,26}$, and BBB leakage has also been reported in human postmortem samples of individuals with $\mathrm{SZ}^{8}$ or $22 \mathrm{qDS}+\mathrm{SZ}^{23}$. We performed $\mathrm{RT}$-qPCR in BMECs and found that CLDN5 mRNA levels were significantly lower in SZ and BD BMECs compared to HC BMECs, but there was no difference in mRNA levels of OCLN or TJP1 (Fig. 1g). Protein levels of CLDN5 and OCLN, but not TJP1, were reduced in SZ BMECs when compared to HC and BD BMECs (Fig. 1h). To determine the cellular attributes that accompany the decreased levels of these tight junction proteins in $\mathrm{SZ}$, we examined morphological features relevant to BBB integrity by quantifying the length (contiguous junctional area), width (health of junctional area), and total intensity of OCLN and CLDN5 in the $\mathrm{BMECs}^{20}$. Both the intensity and width of CLDN5 were significantly reduced in SZ compared to HC (Fig. $1 \mathrm{i}$ and $1 \mathrm{j}$ ), showing a correlation between reduced CLDN5 expression and aberrant BBB-related morphological attributes in SZ. Additionally, OCLN width and length were reduced in SZ and BD BMECS compared to HC BMECs (Fig. $1 \mathrm{i}$ and $1 \mathrm{j}$ ). These results show that that reduced CLDN5 results in morphologies consistent with impaired tight junctions in the SZ BMECs.

BBB deficit subtypes in psychosis. Subtypes of psychosis defined by biosignatures have been pivotal in the pathophysiological understanding of a disorder that is solely defined by clinical syndromes ${ }^{28,29}$. We had previously identified a high inflammatory subtype of psychosis in chronic and first episode psychosis patients using peripheral markers of inflammation and vascular disruption. Studies of postmortem tissue $^{7,30}$, cerebral spinal fluid ${ }^{31}$, peripheral markers ${ }^{32}$, and in vivo brain imaging ${ }^{16,33}$ suggest that BBB disruption exists is $\mathrm{SZ}$ and/or BD and that it can be identified in a subgroup of individuals with psychosis2/11/2022 4:32:00 PM. In order to identify patient subgroups with significantly higher levels of BBB (peak TEER) dysfunction, an outlier analysis was performed (based on the mean minus two standard deviations) in $\mathrm{HC}$ individuals and used to stratify the $\mathrm{SZ}$ and BD groups. Using this method, three of the eight psychosis patients were identified as outliers (peak TEER $<1000 \Omega \times \mathrm{cm}^{2}$ ). The outlier group consisted of 2 SZ and 1 BD patients making up 37.5\% of the sample and were labelled the "BBB-deficit" group. The psychosis group with better BBB function included 2 SZ and 3 BD patients making up $62.5 \%$ of the sample and were labelled "BBB-nondeficit" group. The BBB-deficit group exhibited more extensive BBB disruption as indicated by extremely low TEER values across 6 days of culture (Supplementary Fig. 1b; Fig. 2a) and an absence of day 2 TEER peak (Fig. 2b) compared to HC and BBBnondeficit groups. The BBB-deficit group also had greater permeability to $0.1 \mathrm{kDa}, 4 \mathrm{kDa}$, and $10 \mathrm{kDa}$ compared to $\mathrm{HC}$ individuals and BBB-nondeficit patients (Fig. 2c). Furthermore, analysis of tight junction proteins revealed a reduction in CLDN5 mRNA expression in both the BBB-deficit and the BBB-nondeficit groups (Fig.2d and e), but there was no difference at the MRNA level for OCLN or TJP1 and no notable change in tight junction protein levels across the groups. However, using immunostaining techniques, we found that CLDN5 and OCLN intensity, width, and length were significantly reduced in BBB-deficit BMECs compared to HC BMECs (Fig. $2 \mathrm{f}$ and g). Compared to the BBB-nondeficit group, BBB-deficit BMECs had lower CLDN5 intensity and width, as well as lower OCLN intensity (Fig. $2 f$ and g). Lastly, the BBB-nondeficit BMECs showed lower CLDN5 and OCLN width when compared to HC BMECs (Fig. $2 f$ and g). Similar to existing clinical studies ${ }^{7,15-17,30,31}$, our results demonstrate there is a BBB-deficit subgroup in 
psychotic disorders characterized by more extensive BBB disruption as evidenced by decreased TEER, increased permeability, lower CLDN5 intensity, and aberrant tight junctional morphology.

MMP1 impairs BBB function. Studies suggest that peripheral inflammation may be causally related to psychosis $^{35}$, but the source of inflammation is currently unknown. BMECs are a potential source of

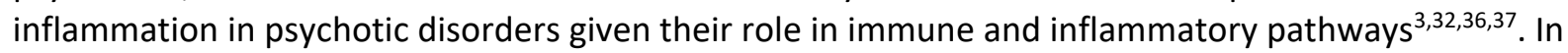
order to test the hypothesis that BMECs secrete factors that can alter BBB function, we performed cross-culture experiments to determine: 1 ) whether secreted factors from $\mathrm{HC} B M E C$ s can improve $B B B$ function (i.e., improve TEER) in BBB-deficit BMECs and 2) whether secreted factors from BBB-deficit BMECs can impair BBB function in HC BMECs. For the first scenario, HC BMECs were seeded in the bottom chamber of a transwell plate and BBB-deficit or -nondeficit BMECs were seeded in the upper chamber (Fig. 3a). Using this experimental design, we observed that exposure to media from HC BMECs improved peak TEER values in 2 out of the 3 BBB-deficit lines (Fig. 3b) and this effect was sustained over 6 days (Supplementary Fig. 2a). In the second scenario (HC BMECs seeded on top and BBB-deficit or nondeficit BMECs seeded on bottom), we observed a decrease in TEER values for the HC BMECs on day 2 (Fig. 3a) and a delayed peak on day 3 with a decreasing trend over time (Supplementary Fig. 2a). These data provide evidence that BMECs secrete factors that impact BBB function as evidenced by media from $\mathrm{HC} B M E C$ s rescuing $B B B$ function in BBB-deficit BMECs while media from BBB-deficit BMECs impaired BBB function in HC BMECs.

BMECs are known to release several cytokines, chemokines, and growth factors in response to a variety of environmental stimuli, and as part of BBB homeostasis ${ }^{38-41}$. Prior studies of peripheral inflammation in psychosis have linked inflammation with the development of psychosis ${ }^{35}$. We performed a targeted proteomic screen of 48 markers related to inflammation ${ }^{39,41}$ in BMEC-conditioned media. Of 48 markers, only 27 proteins had sufficient data for analysis that passed quality control metrics (Supplementary Table 2). CXCL12 levels were increased in conditioned media from SZ BMECs when compared to HC and BD individuals (Supplementary Fig. 2b and c). Conditioned media from BBB-deficit BMECs showed increased CXCL8 and CCL2 levels when compared to conditioned media from HC and BBB-nondeficit BMECs, while CCL19 and MMP1 levels were higher in the BBB-deficit group compared to the BBBnondeficit group (Fig. 3c and d). Inflammatory marker topology showed overall greater inflammatory/chemokine activation in BMECs in SZ and BD when compared to HC (Supplementary Fig. 2d). To determine whether CCL19, CXCL8, CCL2 or MMP1 were related to BBB phenotypes (TEER, cadaverine, dextran, transferrin, CLDN5 expression, CLDN5 protein level), we performed a correlational analysis which demonstrated that higher levels of MMP1 were associated with reduced TEER $(r=-0.79, p$ $\leq 0.01$ ) and CLDN5 protein levels ( $r=-0.61, p \leq 0.05$ ), as well as increased permeability to cadaverine 0.1 $\mathrm{kDa}(r=0.64, p \leq 0.05)$ across the whole study sample (Fig. 3e). In addition, higher levels of CXCL8 and CCL2 were associated with reduced TEER and higher CCL19 level was associated with increased permeability to dextran $10 \mathrm{kDa}$ (Fig. 3e). These data suggest that MMP1 may be responsible for the BBB deficits observed in a subgroup of patients with SZ or BD.

In BMECs, matrix metallopeptidase (MMP) proteins regulate vascular remodeling through degradation of the extracellular matrix ${ }^{38}$. MMP1 cleaves extracellular components such as collagen, gelatin, and laminin $^{42}$. At the same time, MMP1 can also activate other MMPs such as MMP2 and MMP9, which in concert can reduce expression levels of tight junction proteins such as TJP1, OCLN, or CLDN5 ${ }^{43}$. Moreover, TNF $\alpha$ and interleukin 6 (IL-6) are pro-inflammatory cytokines that have been directly linked to increased MMP1 levels and can synergistically modulate MMP1 expression ${ }^{44}$. To determine whether MMP1 was differentially expressed in BMECs between groups, we quantified mRNA levels of MMP1 and found no changes in MMP1 expression between diagnostic (Supplementary Fig. 2e) or BBB-deficit 
categories (Fig. 3f). MMP1 protein levels were increased in the SZ (Supplementary Fig. 2e) and BBBdeficit group (Fig. $3 \mathrm{~g}$ ) compared to HC or BD groups, but this difference was not significant. Since MMP1 is initially released from the cytosol in an inactive form and is subsequently activated by proteolytic cleavage ${ }^{45}$, we quantified MMP1 activity in the BMEC culture media. MMP1 activity was significantly higher in the BBB-deficit group compared to HC (Fig. 3h), but there was no significant difference between the diagnostic categories (Supplementary Fig. 2f). Furthermore, while increased MMP1 activity was associated with reduced TEER ( $r=-0.477)$, CLDN5 expression $(r=-0.349)$ and CLDN5 protein levels $(r=-$ 0.359), the effect was not significant (data not shown). These data suggest that factors affecting MMP1 release and activation in the BBB-deficit group may be responsible the aberrant BBB function.

Inhibition of MMP1 restores BBB function. Since increased MMP1 activity was observed in the BBBdeficit groups, we sought to test whether inhibition of MMP1 can restore BBB function in the BBB-deficit group. Piroxicam is a non-steroidal anti-inflammatory drug that broadly inhibits several MMPs but is highly selective for MMP1 compared to other MMPs ${ }^{46}$. Infliximab is an immunosuppressive drug that specifically targets $\mathrm{TNF}^{47}$ and has been shown to decrease MMP1 levels, endothelial cell necroptosis, and to prevent BBB leakage ${ }^{48}$. BMECs from BBB-deficit and $\mathrm{HC}$ lines treated with infliximab or piroxicam demonstrated a persistent improvement in TEER values over 6 days, but no such effect was seen in BMECs from the BBB-nondeficit groups (Fig. 4a). BMECs from the BBB-deficit group demonstrated an improvement in peak TEER when treated with infliximab (170\%) or piroxicam (166\%) when compared to untreated conditions (Fig. 4b). HC BMECs also demonstrated an increase in peak TEER with piroxicam (145\%), but not with infliximab (Fig. 4b). In order to confirm that these drugs are engaging the target in BBB-deficit BMECs, we measured MMP1 activity in the presence or absence of infliximab or piroxicam. We found a trend towards reduction in MMP1 activity when BBB-deficit BMEC were treated with piroxicam (78\%) as opposed to infliximab (55\%) thought the difference was not statistically significant (Fig. 4c). Additionally, both drugs increased CLDN5 gene expression, but had no effect on CLDN5 intensity in BBB-deficit BMECs (Fig. 4d and Supplementary Fig. 3). Taken together, these data suggest that inhibition of TNF $\alpha$ or MMP1 leads to specific effects and modulates BBB-related features in BBBdeficit lines, but not in BBB-nondeficit lines.

\section{Discussion}

In this study, we utilized in vitro models of BBB function using iPSC-derived BMECs from individuals with $\mathrm{SZ}, \mathrm{BD}$ and $\mathrm{HC}$ to demonstrate that BBB properties (integrity and permeability) and molecular markers (CLDN5 and OCLN) are most impaired in BMECS from SZ patients compared to BMECs from HC individuals, with BMECs from BD patients showing an intermediate phenotype. Notably, we identified a subgroup of patients with SZ or BD that demonstrated substantial impairments in BBB function. BMECs from this BBB-deficit group showed reduced barrier integrity and increased permeability as well as decreased CLDN5 and OCLN levels compared to BMECs HC and BBB-nondeficit individuals. Cross-culture experiments suggested that factors released by BBB-deficit BMECs can impair BBB function in $\mathrm{HC}$ $B M E C s$, and that the secretome from HC BMECs can restore BBB function in BBB-deficit BMECs. Proteomic screening of BMEC-conditioned medium showed greater levels of active MMP1 in BBB-deficit lines. Higher level of MMP1 activity was subsequently found to be associated with reduced barrier integrity, increased permeability, and decreased CLDN5 protein levels. Pharmacologic inhibition of MMP1 with piroxicam and inhibition of TNF $\alpha$, an upstream regulator of MMP1, with infliximab, restored BBB function in BBB-deficit BMECs.

Our experimental data using human iPSC-derived BMECs offer direct evidence for intrinsic deficits in BMECs in SZ that potentially explain neuroanatomical changes described in psychotic disorders. Ultrastructural changes in brain endothelial cells have been described in aborted embryos from mothers 
with $\mathrm{SZ}^{49}$, as well as in postmortem tissue from SZ patients ${ }^{50}$, including increased endothelial surface area, cytoplasmic vacuolization, granulation, thickening of the basal lamina, and plasma membrane disruption. Additionally, transcriptional profiling of brain endothelial cells in SZ patients have identified differences in expression of genes related to immune, inflammation, angiogenesis, and cell adhesion ${ }^{8,51}$. While these studies are of major importance and had implicated BMEC dysfunction in SZ, they did not delineate the specific effects of brain endothelial cells and instead examined the brain as a whole. Some of these studies have also described changes related to astrocyte, microglia, oligodendrocyte, or neurons, making it difficult to interpret the cellular source of BBB dysfunction in SZ pathogenesis ${ }^{50,52,53}$. In an effort to elucidate this complex interplay between the different cell types, a study using human iPSC-derived neural stem cells demonstrated that the secretion of several angiogenic factors from SZ patients reduced endothelial cell migration and angiogenesis, suggesting a dysregulated cross-talk between neural stem cells and endothelial cells during neurovascular development in $\mathrm{SZ}^{54}$. Conversely, two studies examining human iPSC-derived BMECs in patients with 22qDS and SZ demonstrated showed the presence of impaired barrier integrity ${ }^{23,55}$, a phenotype that had been demonstrated in animal models of $22 \mathrm{qDS}^{26}$, suggesting a primary role of BMECs in BBB dysfunction in SZ pathogenesis.

Our CLDN5 results are consistent recapitulate findings of decreased CLDN5 levels in postmortem studies of patient with $\mathrm{SZ}^{8}$. Higher levels of IgA against CLDN5 have been described in the blood of patients with SZ, suggesting the presence of paracellular and BMEC disruption ${ }^{56}$. Furthermore, 22qDS syndrome, which includes haploinsufficiency for CLDN5, is a well-established risk factor for the development of SZ and $\mathrm{BD}^{26}$. A genetic variant of CLDN5 ( $r$ 10314) has been associated with a $50 \%$ reduction in BMECspecific CLDN5 expression and is weakly associated with SZ or BD risk ${ }^{26,57-62}$. Mouse models of CLDN5 knockdown show the presence of learning and memory impairments, sensorimotor gating deficits, and in vivo BBB leakage, further supporting the notion that CLDN5 plays a role in BBB function and SZ phenotypes ${ }^{26}$. Rodent studies also show a dose-dependent increase in CLDN5 levels with exposure to lithium or antipsychotics, suggesting that the therapeutic effects of these drugs may involve their effects on the neurovasculature ${ }^{26}$.

The identification of a BBB subtype (37.5\%) in this study is consistent with prior evidence in human studies that had identified subgroups of patients with elevated peripheral inflammation and/or BBB disruption. There are several studies, including our own, which show the presence of peripheral inflammation in in 30-50\% of patients with chronic psychosis ${ }^{17,63}$ and in first-episode schizophrenia ${ }^{15}$ which was associated with in vivo evidence of BBB disruption ${ }^{15,17}$. Additionally, studies show bloodcerebrospinal fluid barrier (BCSFB) disruption in 22-41\% of patients with SZ and/or BD disorder ${ }^{31,64}$. This work was further expanded by our group and others demonstrating enlarged choroid plexus volume, a key BCSFB structure, in chronic or first-episode psychosis patients, and that greater choroid plexus volume was associated with greater IL-6 levels ${ }^{65}$ or allostatic load ${ }^{66}$, suggesting a complex interplay between systemic inflammation and BBB breakdown. BBB subtypes have also been described using lymphocyte $(41 \%)^{67}$ and perivascular macrophage $(40 \%)^{7}$ density in patients with SZ, the latter of which was found in a subgroup of patients of high cortical inflammation. While these studies have expanded our understanding of the role of inflammatory-immune-BBB nexus in SZ and BD, they do not point to specific cellular mechanisms priming this response.

In addition to demonstrating that intrinsic properties BMECs may be the source of BBB deficits in a subgroup of patients with $\mathrm{SZ}$ or $\mathrm{BD}$, we also show evidence that MMP1 is involved in mediating BBB disruption in these lines. MMP1 has not been previously studied or implicated in SZ or BD. However, MMP1 has been linked to MMP9 activation and MMP1 can be regulated by TNF $\alpha$, both of which have been shown to play a role in SZ and/or BD pathogenesis ${ }^{68-70}$. While it is well established that MMP1 can 
reduce expression of $\mathrm{CLDN}^{43}$, which could provide a mechanistic link for understanding BBB in BD and $S Z$, this effect was not observed in our study. With this mechanism in mind, we examined the effects of infliximab (TNF $\alpha$ biologic) and piroxicam (MMP1 inhibitor) which were found to partially rescue BBB function in BBB-deficit BMECs. While infliximab has not been used for the adjunctive treatment of SZ or $B D$, a recent review examining the association between antibody immunotherapy for immune/inflammatory disorders and psychosis, found that 4 out of 8 antibody therapies suppressing TNF $\alpha$ (including infliximab) correlated with a decreased risk of psychosis ${ }^{71}$. However, cases describing acute psychosis after the administration of TNF $\alpha$ antibody therapy have also been reported ${ }^{72,73}$. Piroxicam has not been used as an adjunctive treatment for SZ or BD, but studies of non-selective COX inhibitor (aspirin) and selective COX-2 inhibitor (celecoxib) in $\mathrm{SZ}^{74,75}$ have reported symptomatic improvement in SZ patients, with the greatest benefit during a first episode of psychosis ${ }^{76}$. These studies demonstrate the potential clinical importance of using drugs that target TNF $\alpha / M M P 1$ for the treatment of patients with SZ or BD. Our study provides a scientific basis for the hypothesis that patients in the BBB-deficit subgroup may be more likely to respond to therapies targeted at TNF $\alpha$ or MMP1.

Taken together, our work shows the existence of BBB-deficit subgroup in SZ and BD and offers a mechanistic link between inherent BMEC dysfunction and peripheral inflammatory activation. Our findings provide a strong rationale using specific pharmacological or biological interventions targeting TNF $\alpha$ or MMP1 mediated BBB disruption as potential therapeutic approaches.

\section{Data availability}

Additional data supporting the findings of this study are available from the corresponding authors upon reasonable request.

\section{Code availability}

Any code used to support the findings of this study is available from the corresponding authors upon reasonable request.

\section{References}

1. Murray, C. J. L. et al. Disability-adjusted life years (DALYs) for 291 diseases and injuries in 21 regions, 1990-2010: a systematic analysis for the Global Burden of Disease Study 2010.

The Lancet 380, 2197-2223 (2012).

2. Martin, J., Taylor, M. J. \& Lichtenstein, P. Assessing the evidence for shared genetic risks across psychiatric disorders and traits. Psychol. Med. 48, 1759-1774 (2018).

3. Pong, S., Karmacharya, R., Sofman, M., Bishop, J. R. \& Lizano, P. The Role of Brain Microvascular Endothelial Cell and Blood-Brain Barrier Dysfunction in Schizophrenia. Complex Psychiatry 6, 30-46 (2020). 
4. Liebner, S. et al. Functional morphology of the blood-brain barrier in health and disease. Acta Neuropathol. (Berl.) 135, 311-336 (2018).

5. Hwang, Y. et al. Gene expression profiling by mRNA sequencing reveals increased expression of immune/inflammation-related genes in the hippocampus of individuals with schizophrenia. Transl. Psychiatry 3, e321-e321 (2013).

6. Greene, C., Hanley, N. \& Campbell, M. Claudin-5: gatekeeper of neurological function. Fluids Barriers CNS 16, 3 (2019).

7. Cai, H. Q. et al. Increased macrophages and changed brain endothelial cell gene expression in the frontal cortex of people with schizophrenia displaying inflammation. Mol. Psychiatry (2018) doi:10.1038/s41380-018-0235-x.

8. Katsel, P., Roussos, P., Pletnikov, M. \& Haroutunian, V. Microvascular anomaly conditions in psychiatric disease. Schizophrenia - angiogenesis connection. Neurosci. Biobehav. Rev. 77, 327-339 (2017).

9. de Klerk, O. L. et al. Regional increase in P-glycoprotein function in the blood-brain barrier of patients with chronic schizophrenia: Psychiatry Res. Neuroimaging 183, 151-156 (2010).

10. Hoosain, F. G. et al. Bypassing P-Glycoprotein Drug Efflux Mechanisms: Possible Applications in Pharmacoresistant Schizophrenia Therapy. BioMed Res. Int. 2015, 1-21 (2015).

11. Kimchi-Sarfaty, C. et al. A 'Silent' Polymorphism in the MDR1 Gene Changes Substrate Specificity. Science 315, 525-528 (2007).

12. Martínez-Magaña, J. J. et al. Exploratory Analysis of Rare and Novel Variants in Mexican Patients Diagnosed with Schizophrenia and Dementia. Rev. Investig. Cl nica 71, 1879 (2019). 
13. Girard, S. L. et al. Increased exonic de novo mutation rate in individuals with schizophrenia. Nat. Genet. 43, 860-863 (2011).

14. Najjar, S. et al. Neurovascular Unit Dysfunction and Blood-Brain Barrier Hyperpermeability Contribute to Schizophrenia Neurobiology: A Theoretical Integration of Clinical and Experimental Evidence. Front. Psychiatry 8, 83 (2017).

15. Hoang, D. et al. Inflammatory Subtypes in Antipsychotic-Naïve First-Episode Schizophrenia are Associated with Altered Brain Morphology and Topological Organization. Brain. Behav. Immun. 100, 297-308 (2022).

16. Kamintsky, L. et al. Blood-brain barrier imaging as a potential biomarker for bipolar disorder progression. NeuroImage Clin. 102049 (2019) doi:10.1016/j.nicl.2019.102049.

17. Lizano, P. et al. Multivariate relationships between peripheral inflammatory marker subtypes and cognitive and brain structural measures in psychosis. Mol. Psychiatry 26, 3430-3443 (2021).

18. Hollmann, E. K. et al. Accelerated differentiation of human induced pluripotent stem cells to blood-brain barrier endothelial cells. Fluids Barriers CNS 14, 9 (2017).

19. Pong, S., Lizano, P. \& Karmacharya, R. Derivation, Expansion, Cryopreservation and Characterization of Brain Microvascular Endothelial Cells from Human Induced Pluripotent Stem Cells. J. Vis. Exp. 61629 (2020) doi:10.3791/61629.

20. Neal, E. H. et al. A Simplified, Fully Defined Differentiation Scheme for Producing BloodBrain Barrier Endothelial Cells from Human iPSCs. Stem Cell Rep. 12, 1380-1388 (2019).

21. Lippmann, E. S. et al. Derivation of blood-brain barrier endothelial cells from human pluripotent stem cells. Nat. Biotechnol. 30, 783-791 (2012). 
22. Lim, R. G. et al. Huntington's Disease iPSC-Derived Brain Microvascular Endothelial Cells Reveal WNT-Mediated Angiogenic and Blood-Brain Barrier Deficits. Cell Rep. 19, 13651377 (2017).

23. Crockett, A. M. et al. Disruption of the blood-brain barrier in 22q11.2 deletion syndrome. Brain 144, 1351-1360 (2021).

24. Lizano, P. L. et al. Angiogenic and immune signatures in plasma of young relatives at familial high-risk for psychosis and first-episode patients: A preliminary study. Schizophr. Res. 170, 115-122 (2016).

25. Blanchard, J. W. et al. Reconstruction of the human blood--brain barrier in vitro reveals a pathogenic mechanism of APOE4 in pericytes. Nat. Med. 26, 952-963 (2020).

26. Greene, C. et al. Dose-dependent expression of claudin-5 is a modifying factor in schizophrenia. Mol. Psychiatry 23, 2156-2166 (2018).

27. McCandless, S. E., Scott, J. A. \& Robin, N. H. Deletion 22q11: A Newly Recognized Cause of Behavioral and Psychiatric Disorders. Arch. Pediatr. Adolesc. Med. 152, (1998).

28. Tamminga, C. A. et al. Biotyping in psychosis: using multiple computational approaches with one data set. Neuropsychopharmacology 46, 143-155 (2021).

29. Clementz, B. A. et al. Identification of Distinct Psychosis Biotypes Using Brain-Based Biomarkers. Am. J. Psychiatry 173, 373-384 (2016).

30. Busse, S. et al. Different distribution patterns of lymphocytes and microglia in the hippocampus of patients with residual versus paranoid schizophrenia: Further evidence for disease course-related immune alterations? Brain. Behav. Immun. 26, 1273-1279 (2012). 
31. Bechter, K. et al. Cerebrospinal fluid analysis in affective and schizophrenic spectrum disorders: Identification of subgroups with immune responses and blood-CSF barrier dysfunction. J. Psychiatr. Res. 44, 321-330 (2010).

32. Futtrup, J. et al. Blood-brain barrier pathology in patients with severe mental disorders: a systematic review and meta-analysis of biomarkers in case-control studies. Brain Behav. Immun. - Health 6, 100102 (2020).

33. Kelly, S. et al. White matter microstructure across brain-based biotypes for psychosis findings from the bipolar-schizophrenia network for intermediate phenotypes. Psychiatry Res. Neuroimaging 308, 111234 (2021).

34. Bishop, J. R., Zhang, L. \& Lizano, P. Inflammation subtypes and translating inflammationrelated genetic findings in schizophrenia and related psychoses: a perspective on pathways for treatment stratification and novel therapies. Harv. Rev. Psychiatry 30, (2022).

35. Kappelmann, N., Perry, B. I. \& Khandaker, G. M. Prenatal and Childhood ImmunoMetabolic Risk Factors for Adult Depression and Psychosis. Harv. Rev. Psychiatry 30,.

36. Afridi, R., Seol, S., Kang, H. J. \& Suk, K. Brain-immune interactions in neuropsychiatric disorders: Lessons from transcriptome studies for molecular targeting. Biochem. Pharmacol. 188, 114532 (2021).

37. Comer, A. L., Carrier, M., Tremblay, M.-È. \& Cruz-Martín, A. The Inflamed Brain in Schizophrenia: The Convergence of Genetic and Environmental Risk Factors That Lead to Uncontrolled Neuroinflammation. Front. Cell. Neurosci. 14, 274 (2020).

38. Suzuki, Y., Nagai, N. \& Umemura, K. A Review of the Mechanisms of Blood-Brain Barrier Permeability by Tissue-Type Plasminogen Activator Treatment for Cerebral Ischemia. Front. Cell. Neurosci. 10, (2016). 
39. O’Carroll, S. J. et al. Pro-inflammatory TNF $\alpha$ and IL-1 $\beta$ differentially regulate the inflammatory phenotype of brain microvascular endothelial cells. J. Neuroinflammation 12, $131(2015)$.

40. Yu, Q., Tao, H., Wang, X. \& Li, M. Targeting brain microvascular endothelial cells: a therapeutic approach to neuroprotection against stroke. Neural Regen. Res. 10, 1882 (2015).

41. Miao, Z. et al. VEGF Increases Paracellular Permeability in Brain Endothelial Cells via Upregulation of EphA2: VEGF Induces EphA2 Expression for Permeability. Anat. Rec. 297, 964-972 (2014).

42. Inflammation in Heart Failure. (Elsevier, 2015). doi:10.1016/C2013-0-13049-8.

43. Rempe, R. G. et al. Matrix Metalloproteinase-Mediated Blood-Brain Barrier Dysfunction in Epilepsy. J. Neurosci. 38, 4301-4315 (2018).

44. Du, G. et al. Induction of matrix metalloproteinase-1 by tumor necrosis factor- $\alpha$ is mediated by interleukin-6 in cultured fibroblasts of keratoconus. Exp. Biol. Med. 241, 2033-2041 (2016).

45. Skin aging handbook: an integrated approach to biochemistry and product development. (William Andrew, 2008).

46. Leite, S. R. de A. Inhibitors of human collagenase, MMP1. Eclética Quím. 34, 87-102 (2009).

47. Shealy, D. J. et al. Characterization of golimumab, a human monoclonal antibody specific for human tumor necrosis factor $\alpha$. mAbs 2, 428-439 (2010).

48. Chen, A.-Q. et al. Microglia-derived TNF- $\alpha$ mediates endothelial necroptosis aggravating blood brain-barrier disruption after ischemic stroke. Cell Death Dis. 10, 487 (2019). 
49. Orlovskaia, D. D. \& Solov'eva, Z. V. [Changes in the ultra-fine structure of capillaries of the embryonic brain in the presence of schizophrenia in the mother]. Zhurnal Nevropatol. Psikhiatrii Im. SS Korsakova Mosc. Russ. 1952 76, 1043-1046 (1976).

50. Uranova, N. A. et al. Ultrastructural damage of capillaries in the neocortex in schizophrenia. World J. Biol. Psychiatry 11, 567-578 (2010).

51. Harris, L. W. et al. The Cerebral Microvasculature in Schizophrenia: A Laser Capture Microdissection Study. PLoS ONE 3, e3964 (2008).

52. Saetre, P. et al. Inflammation-related genes up-regulated in schizophrenia brains. BMC Psychiatry 7, 46 (2007).

53. Orlovskaia, D. D., Solov'eva, Z. V. \& Zimina, I. S. [Ultrastructural features of brain cells from the embryos of schizophrenic women]. Zhurnal Nevropatol. Psikhiatrii Im. SS Korsakova Mosc. Russ. 1952 75, 1041-1044 (1975).

54. Casas, B. S. et al. hiPSC-derived neural stem cells from patients with schizophrenia induce an impaired angiogenesis. Transl. Psychiatry 8, 48 (2018).

55. Li, Y. et al. Investigation of Neurodevelopmental Deficits of 22 q11.2 Deletion Syndrome with a Patient-iPSC-Derived Blood-Brain Barrier Model. Cells 10, 2576 (2021).

56. Maes, M., Sirivichayakul, S., Kanchanatawan, B. \& Vodjani, A. Breakdown of the Paracellular Tight and Adherens Junctions in the Gut and Blood Brain Barrier and Damage to the Vascular Barrier in Patients with Deficit Schizophrenia. Neurotox. Res. 36, 306-322 (2019).

57. Sun, Z.-Y. et al. The CLDN5 locus may be involved in the vulnerability to schizophrenia. Eur. Psychiatry 19, 354-357 (2004). 
58. Ye, L. et al. Further study of a genetic association between the CLDN5 locus and schizophrenia. Schizophr. Res. 75, 139-141 (2005).

59. Wu, N. et al. A weak association of the CLDN5 locus with schizophrenia in Chinese casecontrol samples. Psychiatry Res. 178, 223 (2010).

60. Omidinia, E., Mazar, F. M. \& Shahamati, P. Polymorphism of the CLDN5 gene and Schizophrenia in an Iranian Population. 43, 5 (2014).

61. Schizophrenia Working Group of the Psychiatric Genomics Consortium. Biological insights from 108 schizophrenia-associated genetic loci. Nature 511, 421-427 (2014).

62. Psychiatric GWAS Consortium Bipolar Disorder Working Group. Large-scale genome-wide association analysis of bipolar disorder identifies a new susceptibility locus near ODZ4. Nat. Genet. 43, 977-983 (2011).

63. Fillman, S. G., Cloonan, N., Miller, L. C. \& Weickert, C. S. Markers of inflammation in the prefrontal cortex of individuals with schizophrenia. Mol. Psychiatry 18, 133-133 (2013).

64. Müller, N. Immunoglobulin and albumin content of cerebrospinal fluid in schizophrenic patients: relationship to negative symptomatology. Schizophr. Res. 14, 223-228 (1995).

65. Lizano, P. et al. Association of Choroid Plexus Enlargement With Cognitive, Inflammatory, and Structural Phenotypes Across the Psychosis Spectrum. Am. J. Psychiatry 176, 564-572 (2019).

66. Zhou, Y.-F. et al. Choroid Plexus Enlargement and Allostatic Load in Schizophrenia. Schizophr. Bull. 46, 722-731 (2020).

67. Schlaaff, K. et al. Increased densities of T and B lymphocytes indicate neuroinflammation in subgroups of schizophrenia and mood disorder patients. Brain. Behav. Immun. 88, 497-506 (2020). 
68. Bitanihirwe, B. K. Y. \& Woo, T.-U. W. A conceptualized model linking matrix metalloproteinase-9 to schizophrenia pathogenesis. Schizophr. Res. (2020) doi:10.1016/j.schres.2019.12.015.

69. Reininghaus, E. Z. et al. Extracellular matrix proteins matrix metallopeptidase 9 (MMP9) and soluble intercellular adhesion molecule 1 (sICAM-1) and correlations with clinical staging in euthymic bipolar disorder. Bipolar Disord. 18, 155-163 (2016).

70. Goldsmith, D. R., Rapaport, M. H. \& Miller, B. J. A meta-analysis of blood cytokine network alterations in psychiatric patients: comparisons between schizophrenia, bipolar disorder and depression. Mol. Psychiatry 21, 1696-1709 (2016).

71. Essali, N., Goldsmith, D. R., Carbone, L. \& Miller, B. J. Psychosis as an adverse effect of monoclonal antibody immunotherapy. Brain. Behav. Immun. 81, 646-649 (2019).

72. Locher, M. R. \& Alam, A. Acute Psychosis in an Adolescent Treated With Infliximab for Crohn's Disease. Prim. Care Companion CNS Disord. (2015) doi:10.4088/PCC.15101781.

73. Thillard, E.-M. et al. Psychiatric Adverse Events Associated With Infliximab: A Cohort Study From the French Nationwide Discharge Abstract Database. Front. Pharmacol. 11, 513 (2020).

74. Mehta, J., Chen, J., Yu, F. \& Li, D. Aspirin inhibits ox-LDL-mediated LOX-1 expression and metalloproteinase-1 in human coronary endothelial cells. Cardiovasc. Res. 64, 243-249 (2004).

75. Tsutsumi, R. et al. Celecoxib inhibits production of MMP and NO via down-regulation of NF- $\kappa \mathrm{B}$ and JNK in a PGE2 independent manner in human articular chondrocytes.

Rheumatol. Int. 28, 727-736 (2008). 
76. Çakici, N., van Beveren, N. J. M., Judge-Hundal, G., Koola, M. M. \& Sommer, I. E. C. An update on the efficacy of anti-inflammatory agents for patients with schizophrenia: a metaanalysis. Psychol. Med. 49, 2307-2319 (2019).

77. Kathuria, A. et al. Synaptic deficits in iPSC-derived cortical interneurons in schizophrenia are mediated by NLGN2 and rescued by N-acetylcysteine. Transl. Psychiatry 9, 321 (2019).

78. Kathuria, A. et al. Transcriptomic Landscape and Functional Characterization of Induced Pluripotent Stem Cell-Derived Cerebral Organoids in Schizophrenia. JAMA Psychiatry (2020) doi:10.1001/jamapsychiatry.2020.0196.

79. Kathuria, A. et al. Transcriptome analysis and functional characterization of cerebral organoids in bipolar disorder. Genome Med. 12, 34 (2020).

80. Li, C. H. \& Lee, C. K. Minimum cross entropy thresholding. Pattern Recognit. 26, 617-625 (1993).

81. Li, C. H. \& Tam, P. K. S. An iterative algorithm for minimum cross entropy thresholding. Pattern Recognit. Lett. 19, 771-776 (1998).

\section{Acknowledgements}

This work was supported by a National Institute of Mental Health Biobehavioral Research Awards for Innovative New Scientists (BRAINS) Award R01MH113858 (to R.K.), the National Institutes of Health Award KL2 TR002542 (PL), the Stuart T. Hauser Research Training Program in Biological and Social Psychiatry T32 MH 016259 (SS). the National Institute of Mental Health Clinical Scientist Development Award K08MH086846 (to R.K.), the Sydney R Baer Jr Foundation Grant (to P.L.), the One Mind Bipolar Disorders Award (to P.L.), the Doris Duke Charitable Foundation Clinical Scientist Development Award (to R.K.), the Ryan Licht Sang Bipolar Foundation (to R.K.), the Phyllis \& Jerome Lyle Rappaport Foundation (to R.K.), the Harvard Stem Cell Institute (to R.K.) and by Steve Willis and Elissa Freud (to R.K.). We would like to thank Dr. Donna McPhie and Dr. Bruce Cohen for providing fibroblasts that were used to generate iPSCs for this study.

\section{Author contributions}

P.L. conceived the idea. R.K. assisted with study design. P.L. developed/adapted the BMEC protocol and collected pilot data for the study. S.P. performed and analyzed TEER experiment, permeability, RT-qPCR, immunocytochemistry, co-culturing, and MMP1 inhibition assays. P.L. and S.P. analyzed Olink proteomic assays. S.P. and S.S. performed and analyzed western blots. D.B. contributed Matlab code and processed ICC images for tight junction proteins quantification. P.L. and S.P. wrote the first draft of this manuscript. P.L., S.P., S.S., D.B., and R.K. edited and revised the manuscript. 


\section{Competing interests}

None of the other authors declare any competing interests.

\section{Methods}

Cell lines. Human induced pluripotent stem cells (iPSC) were generated from fibroblasts using mRNAbased genetic reprogramming (Cellular Reprogramming Inc.) from age-and sex-matched chronic schizophrenia $(n=4)$, psychotic bipolar I disorder $(n=4)$, and healthy control $(n=4)$ patients, as approved by the Institutional Review Boards (IRB) ${ }^{77-79}$. Two male and two female patient lines were included for each group. All human iPSCs were maintained in xeno-free conditions in NutriStem medium (ReproCell, 01-0005), expressed appropriate pluripotency characteristics, and were found to be karyotypically normal using the KaryoStat Assay ${ }^{77-79}$. Information on these cell lines is provided in Supplementary Table 1.

Differentiation of iPSCs to BMECs. iPSCs were differentiated into BMEC-like cells following a protocol we have described earlier ${ }^{19,20}$. In brief, iPSCs were washed with DPBS and dissociated using Accutase for $5 \mathrm{~min}$ at $37^{\circ} \mathrm{C}$. The cells were collected via centrifugation and seeded onto Geltrex-coated (ThermoFisher Scientific, A1413202) 6-well plates (Corning, 353046) at a density of $146,000 / \mathrm{cm}^{2}$ in NutriStem medium supplemented with $10 \mu \mathrm{M}$ rho-associated kinase (ROCK) inhibitor Y-27632. After 24 hours (day 0), NutriStem medium was changed to E6 medium, which was changed every day for 4 days. On day 4, E6 medium was replaced with human endothelial serum-free medium (hESFMS) (ThermoFisher Scientific, 11111044 ) supplemented with B27 supplement (1:200 dilution), $20 \mathrm{ng} / \mathrm{mL}$ basic fibroblast growth factor (bFGF), and $10 \mu \mathrm{M}$ retinoic acid (RA). On day 6 , cells were washed with DPBS and treated with Accutase for $30-45 \mathrm{~min}$ at $37^{\circ} \mathrm{C}$. These cells were collected by centrifugation and sub-cultured onto plates coated with a mixture of $400 \mu \mathrm{g} / \mathrm{mL}$ collagen IV (COL4, Sigma, C5533-5MG) and $100 \mu \mathrm{g} / \mathrm{mL}$ fibronectin I (FN1, ThermoFisher Scientific, 33016015). Cells were plated using a ratio of 1 well of a 6 -well plate to 3 wells of a 12-well filtered trans-well plate (0.4 $\mu \mathrm{m}$ pores, Fisher Scientific, 07-200-161) and 6 wells of a 24wells plastic-bottom plate (Corning, 353047). On day 7, medium was changed to hESFM with B27 supplement without bFGF and RA.

TEER measurement and analysis. On day 7, TEER was measured using STX2 chopstick electrodes and an EVOM2 voltohmeter (World Precision Instruments) every day for six days. Raw values were corrected for the resistance of an empty transwell filter plate. TEER measurements were performed in triplicate for each well and for each condition. The average value from the blank wells (i.e. empty wells without any cells) were subtracted from the raw values for each condition. The subtracted values (in $\Omega$ ) were multiplied by $1.12 \mathrm{~cm}^{2}$, the surface area of the transwell inserts in the 12-transwell plate and the transformed values were used for the analyses. To identify cell lines with higher level of BBB disruption (BBB-deficit group), we first calculated the mean $\left(3037 \Omega \times \mathrm{cm}^{2}\right.$ ) and the standard deviation (1051 $\Omega \times$ $\mathrm{cm}^{2}$ ) of peak TEER values in the HC group $(\mathrm{n}=4)$. Any peak TEER value that was more than two standard deviations below the mean (i.e. any value $<1000 \Omega \times \mathrm{cm}^{2}$ ) was considered as exhibiting higher level of BBB disruption and was categorized into the BBB-deficit group.

Permeability studies. Cells were plated using a ratio of 1 well of a 6 -well plate to 3 wells of a 12 -well filtered transwell plate (0.4 $\mu \mathrm{m}$ pores, Fisher Scientific, 07-200-161). After $48-72$ hours of subculturing (day 8-9), permeability assays were conducted using $4 \mathrm{kDa}, 10 \mathrm{kDa}$ and $70 \mathrm{kDa}$ dextrans labeled with fluorescein isothiocyanate (Sigma, 46944, FD10S, 46945), transferrin (Thermo Fisher Scientific, T-13342), or Alexa Fluor 555 cadaverine (Thermo Fisher Scientific, a30677) in hESFM supplemented with B27 supplement (1:200 dilution). $1.5 \mathrm{~mL}$ of fresh medium was added to the bottom of the transwell and 0.5 
$\mathrm{mL}$ of dye and medium were added to the top. The wells were then incubated at $37^{\circ} \mathrm{C}$ for 1 hour. Medium from the bottom section of the transwell chamber was collected and analyzed using a 2103 EnVision Multilabel Reader (Perkin Elmer). Averaged values from a blank well, which did not contain any cells, were subtracted from the raw values of the triplicate wells for each condition. The subtracted values (in relative fluorescent unit or RFU) were used for the analyses.

mRNA and protein analysis. RNA was extracted from BMECs that had been subcultured for $48-72$ hours (day 8-9) using trizol to lyse the cells and the RNeasy Mini Kit (Qiagen, 74104) for RNA purification. For RT-PCR, 500ng of RNA was converted to cDNA using High-Capacity CDNA Reverse Transcription Kit (Thermo Fisher Scientific, 4368814). SYBR green (Mangobio, 08-25-00020) assay was run on Roche light cycler 480II. In 364-well plates, each well contained $2 \mu \mathrm{L}$ of Evagreen qPCR Mix, $1 \mu \mathrm{L}$ forward/reverse primers mix, $5 \mu \mathrm{L}$ RNase free water, and $2 \mu \mathrm{L}$ cDNA. For each gene of interest, two technical replicates and three independent biological replicates per cell line were tested. Each gene of interest was normalized to a reference gene, GAPDH. Analysis was performed on Excel spreadsheet and graphs prepared with GraphPad version 8.0. Primers were obtained from KiCqStart ${ }^{\mathrm{TM}}$ Primers (Millipore Sigma) as listed in Supplementary Table 3. For protein extraction, cells were washed with DBPS and lysed with RIPA buffer. Protein concentration was measured with a BCA assay. $10 \mu \mathrm{g}$ of protein of each sample was run on a Criterion TGX Precast gel 4-20\% (BIO-RAD, 5671094) at 120 V for 1.5 hours. Gels were transferred to a Immobilon-P Transfer Membrane (Millipore IPVH00010, pore size: $0.45 \mu \mathrm{m}, \mathrm{PVDF}$ ) and blocked in Odyssey Blocking Buffer (Li-Cor, 927-40,000) for 1 hour at room temperature. Membrane was probed overnight with primary antibodies at $4{ }^{\circ} \mathrm{C}$ (Supplementary Table 4), followed by washes with $1 \mathrm{x}$ TBST, then incubated with secondary antibody using donkey anti-rabbit (Li-Cor 1:10,000, IRDye 680, 925-68073, lot \#C70601-01) or anti-mouse (Li-Cor 1:10,000, IRDye 800, 925-32212, lot \#C70502-03) for 1 hour at room temperature. Images were analyzed using an Odyssey Clx Imaging System (Li-Cor Biosciences) and quantified using Image J.

Immunocytochemistry (ICC). After 48 -72 hours of sub-culturing (day 8-9) on 24-wells plastic-bottom plates (Corning, 353047), cells were washed twice with DPBS. Cells were then fixed with $4 \%$ paraformaldehyde (PFA) and pre-blocked in DPBS with $5 \%$ donkey serum and $0.2 \%$ Triton X-100 for 1 hour at room temperature. Fixed cells were incubated with primary antibodies: rabbit anti-human-TJP1 (1:200), mouse anti-human-CLDN5 (1:200), rabbit anti-human-OCLN (1:200) in DPBS containing 5\% donkey serum overnight at $4^{\circ} \mathrm{C}$. Cells were washed five times with a minimum of 5 minutes per wash using DPBS. Then, they were incubated in secondary antibodies for 1 hour at room temperature. Primary and secondary antibodies are listed in Supplementary Table 4. Cells were then incubated in Hoechst 33342 solution (1:1000), for approximately 1 min before visualizing on IN Cell Analyzer 6000 (GE Healthcare Life Sciences) using fluorescence microcopy settings.

Tight junction protein expression quantification. Raw ICC images were exported in 16-bit TIF format and analyzed using a custom MATLAB script adapted from Neal et al. $2019^{20}$. Six different fields of view were selected for each cell line per condition. Fields of view were selected based on the following criteria: field of few had cells covering the entire field of view, low background noise, and images that were clear and not blurry. In the junction (dsRed and FITC) images, individual pixels were compared to the average pixel intensity within a 10-pixel square radius, resulting in binarized junction images. The binarized images were used to extract tight junctional length, width, and total intensity measures for each tight junction protein (CLDN5 and OCLN) in all cell lines and each field of view. The total number of junction-positive pixels was divided by the number of Hoechst-positive pixels in each field of view as a confluency-normalized metric of junctional density. Individual nuclei were detected using a custom FIJ script that identified DAPI-positive pixels within each image. For this process, raw images were exported 
in a 16-bit TIF format and first converted to 8-bit images. A Kuwahara filter was used with a sampling window width of 11 pixels for smoothing and noise reduction. Images were auto-thresholded using Li's method $^{80,81}$ and converted to a binary mask. A watershed algorithm was used to segment out nuclei, and the number of nuclei were then extracted. Normalized values from each field were averaged and appropriate statistical tests performed to determine differences between each diagnostic group.

Cross-culture assay. Cells were plated using a ratio of 1 well of a 6 -well plate to 3 wells of a 12 -wells filtered transwell plate (0.4 $\mu \mathrm{m}$ pores, Fisher Scientific, 07-200-161). For the first assay, a HC cell line was seeded on the top chamber of the transwell while a BBB-deficit or BBB-nondeficit cell line was seeded on the bottom chamber of the transwell (Figure 3a). In a complementary assay, these conditions were reversed. TEER measurements were collected in triplicate for each well and for each condition and analysis of the TEER measurements were performed as described above.

Olink analysis of BMEC conditioned medium. Conditioned medium was collected from each cell line after 48-72 hours of subculturing and analyzed by Olink Proteomics, Inc. (Watertown, MA) using the Target 48 cytokine platform. Samples were assigned a randomized 4-digit ID number and the assays were run by technicians without any knowledge of the clinical status of the samples. Processing and data quality control were performed by Olink Proteomics. All data were delivered as excel spreadsheet along with the certificate of analysis. Further information on how Olink determines limits of detection (LOD) and sample normalizations can be found online (https://www.olink.com/). Markers with more than $25 \%$ of quantity not sufficient data values were excluded from the analysis. Biomarkers with $<25 \%$ of quantity not sufficient data were imputed to the mean of the samples $(n=12)$ for each respective marker. Among the 48 markers, 27 were detected and selected for further analysis. The full list of biomarkers can be found in Supplementary Table 2. Raw concentration values $(\mathrm{pg} / \mathrm{mL})$ were normalized to the average concentration in the $\mathrm{HC}$ group. Statistical tests were performed as described in the figure legends.

Active MMP1 Fluorokine E assay. Conditioned medium collected from each cell line were examined for levels of active MMP1 protein using standard protocols for the Human Active MMP1 Fluorokine E Kit (R\&D Systems, F1M00). Assays was done in technical duplicates and a standard curve was generated. Concentration of active MMP1 protein in the conditioned medium was calculated based on the standard curve. Any value with a negative concentration was imputed to zero $n g / \mu L(n=7)$.

MMP1 inhibition assay. Infliximab (Sigma Aldrich, CAS\#170277-31-3) and piroxicam (Sigma Aldrich, CAS\# 36322-90-4) were used as TNF $\alpha$ and MMP1 inhibitors, respectively. Stock solution of infliximab was diluted with hESFM to $300 \mathrm{ng} / \mathrm{mL}$ working concentration and piroxicam was diluted to $10 \mu \mathrm{M}$ working concentration. Technical triplicates of the MMP1 inhibition assay were conducted for each condition in three independent biological replicates. Cells were treated with each inhibitor for 24 hours during subculturing before being removed and replenished with fresh medium. TEER measurements, immunocytochemistry, mRNA and protein analysis were conducted as described above.

\section{Statistical Analysis.}

All representative graphs were analyzed and created in $\mathrm{R}$ studio and GraphPad Prism 8. Any statistical analysis such as One-Way or Two-Way ANOVA and post-hoc Tukey test are reported in the figure legends. Correction for multiple comparisons were included when needed. All reported $p$-values are two-sided. Outlier removal was applied when needed using the ROUT method in which Q was set to $0.5 \%$ (i.e. at least $99.5 \%$ are actual outliers). Univariate analysis of variance via Kruskal-Wallis rank sum test was used to identify any moderating effects of sex, iPSCs passage numbers, and cell viabilities (\%) 
on peak TEER measurements across all three biological replicates. Reported chi-squares and p-values from this analysis can be found in the Supplementary Table 5. Bivariate correlations were performed between peripheral markers and BBB phenotypes using Spearman correlations.

Reporting Summary. Further information on research design is available in the Nature Research Reporting Summary linked to this article. 


\section{Figures}
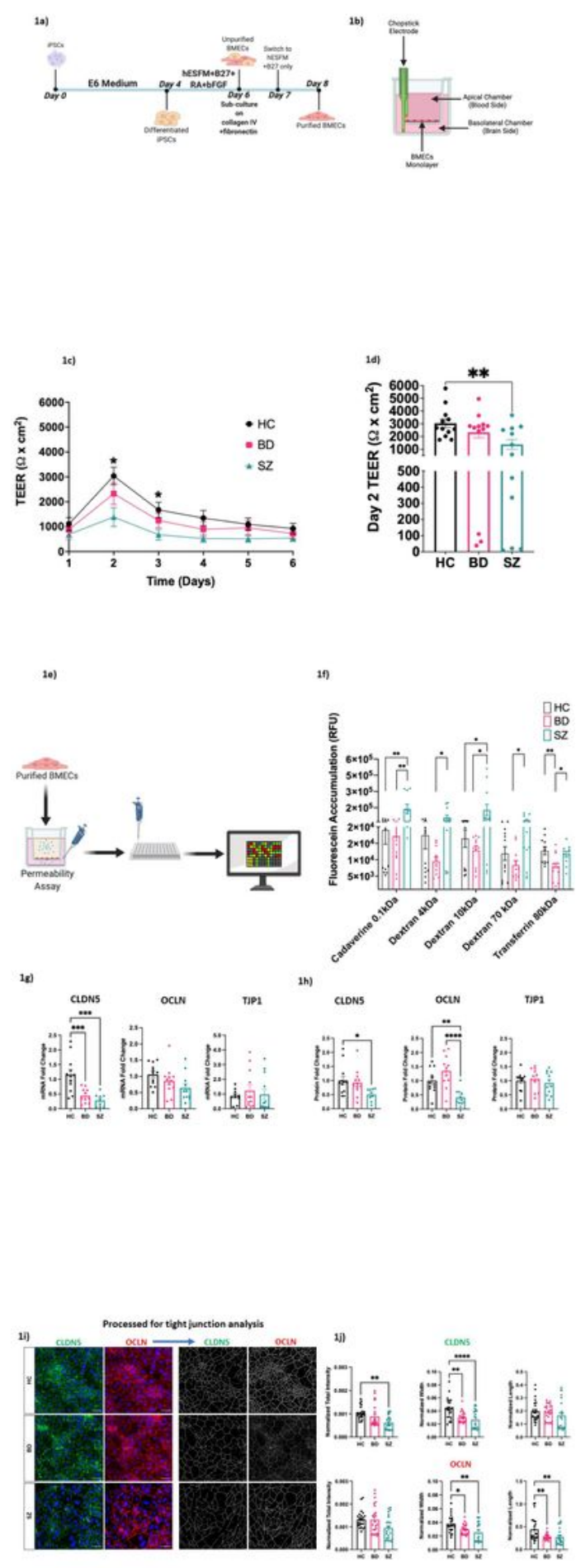

Figure 1

Human iPSC-derived BMECs from SZ patients demonstrate aberrant barrier function in a BBB model. a) Schematic for differentiating human iPSCs into BMECs over an 8-day period. b) Experimental set-up for TEER measurements. c) Average TEER values collected over 6 days for healthy control $(H C, n=4)$, bipolar 
disorder $(B D, n=4)$ and schizophrenia $(S Z, n=4)$ cell lines. Significance was determined by Two-Way ANOVA and post-hoc Tukey multiple comparison test ( ${ }^{*} p=0.011$ comparing $S Z$ to HC on day $2,{ }^{*} p=0.0405$ comparing SZ to HC on day 3). d) Average peak (day 2) TEER values for HC, BD, and SZ BMECs. Significance was determined by One-Way ANOVA and post-hoc Tukey multiple comparison test (**p $=0.0094$, comparing SZ to HC). TEER values were collected for three biological replicates each and shown as mean \pm s.e.m. e) Schematic of permeability assay using fluorescently labeled substrates of varying molecular weight. f) Permeability of fluorescently labeled substrates across a confluent monolayer of BMECs from HC $(n=4), B D(n=4)$, and SZ ( $n=4)$ patients. Permeability assays were conducted at the time of peak TEER values (day 2, Fig.1c). Permeability readings were performed in triplicate using three biological replicates. Permeability values (RFU) were reported using three biological replicates with mean \pm s.e.m. Significance was determined by One-Way ANOVA and post-hoc Tukey multiple comparison tests. Cadaverine $0.1 \mathrm{kDa} * * \mathrm{p}=0.0022$ comparing $\mathrm{SZ}$ to $\mathrm{HC}$ and ${ }^{*} \mathrm{k}=0.0029$ comparing $\mathrm{SZ}$ to $\mathrm{BD}$; dextran $4 \mathrm{kDa}$ ${ }^{*} \mathrm{p}=0.0268$ comparing $\mathrm{SZ}$ to $\mathrm{BD}$; dextran $10 \mathrm{kDa}{ }^{*} \mathrm{p}=0.0358$ comparing $\mathrm{SZ}$ to $\mathrm{HC}$ and ${ }^{*} \mathrm{p}=0.0338$ comparing SZ to $\mathrm{BD}$; dextran $70 \mathrm{kDa}{ }^{*} \mathrm{p}=0.0308$ comparing $\mathrm{SZ}$ to $\mathrm{BD}$; transferrin $80 \mathrm{kDa}{ }^{* \star} \mathrm{p}=0.0087$ comparing $\mathrm{BD}$ to $\mathrm{HC}$ and ${ }^{*} \mathrm{p}=0.0448$ comparing $\mathrm{SZ}$ to $\mathrm{BD}$. 2 outliers were removed using the ROUT method ( $q=0.5$ ). $\mathbf{g}$ ) Normalized mRNA levels in BMECs for TJP1, OCLN, and CLDN5 for HC, BD, and SZ BMECs. mRNA expression for each gene was normalized to the housekeeping gene GAPDH. Each normalized value was then calibrated to the average mRNA expression from the HC group $(n=4)$ using the $\triangle \triangle$ Ct method; RT-qPCR was performed in technical duplicates; each dot represents a biological replicate. Mean \pm s.e.m is shown for each group. Significance was determined by One-Way ANOVA and post-hoc Tukey multiple comparison test ( $* \star p=0.0008$ comparing CLDN5 mRNA expression in BD to HC, $\star \star \star p=0.0001$ comparing CLDN5 mRNA expression in SZ to HC). 7 outliers were removed. $h$ ) Normalized protein levels from $\mathrm{HC}, \mathrm{BD}$, and $\mathrm{SZ}$ groups; each dot represents a biological replicate. Means \pm s.e.m is indicated for each group. Band intensities from western blots were quantified using Fiji by Image $\mathrm{J}$ and normalized to a reference protein (GAPDH). Significance was determined by One-Way ANOVA and posthoc Tukey multiple comparison test ( ${ }^{*} p=0.0405$ comparing CLDN5 protein level in SZ to HC, ${ }^{*} p=0.007$ comparing OLCN protein level in SZ to $\mathrm{HC}$ and ${ }^{* \star \star \star} p<0.0001$ comparing OCLN protein level in SZ to BD). 4 outliers were removed. i) Representative images are shown of immunostaining for CLDN5 (green), OCLN (red), and nuclei (blue) in HC, BD, and SZ patients. Images were processed using Matlab, assessing for fluorescent intensity; scale bar is $50 \mu \mathrm{m}$. j) Normalized total fluorescent intensity, width intensity, and length intensity for OCLN and CLDN5 in BMECs from HC, BD, and SZ lines. Six fields of view for each subject were used for quantification. Raw intensity values were normalized to the total number of nuclei in each respective field of view. Each dot represents one field of view. Mean \pm s.e.m is shown for each group. Significance was determined by One-Way ANOVA and post-hoc Tukey multiple comparison test. ${ }^{\star \star * \star} \mathrm{p}<0.0001$ comparing CLDN5 width in SZ to HC, ${ }^{\star \star} \mathrm{p}=0.0027$ comparing CLDN5 width in BD to HC, ${ }^{* \star} p=0.0012$ comparing CLDN5 total intensity in SZ to HC, ${ }^{*} p=0.0337$ comparing OCLN width in BD to $\mathrm{HC},{ }^{*} \mathrm{p}=0.001$ comparing OCLN width in $\mathrm{SZ}$ to $\mathrm{HC},{ }^{*} \mathrm{p}=0.0082$ comparing OCLN length in BD to HC, ${ }^{\star *} \mathrm{p}=0.0071$ comparing OCLN length in SZ to HC. All reported $\mathrm{p}$-values are two-sided. 

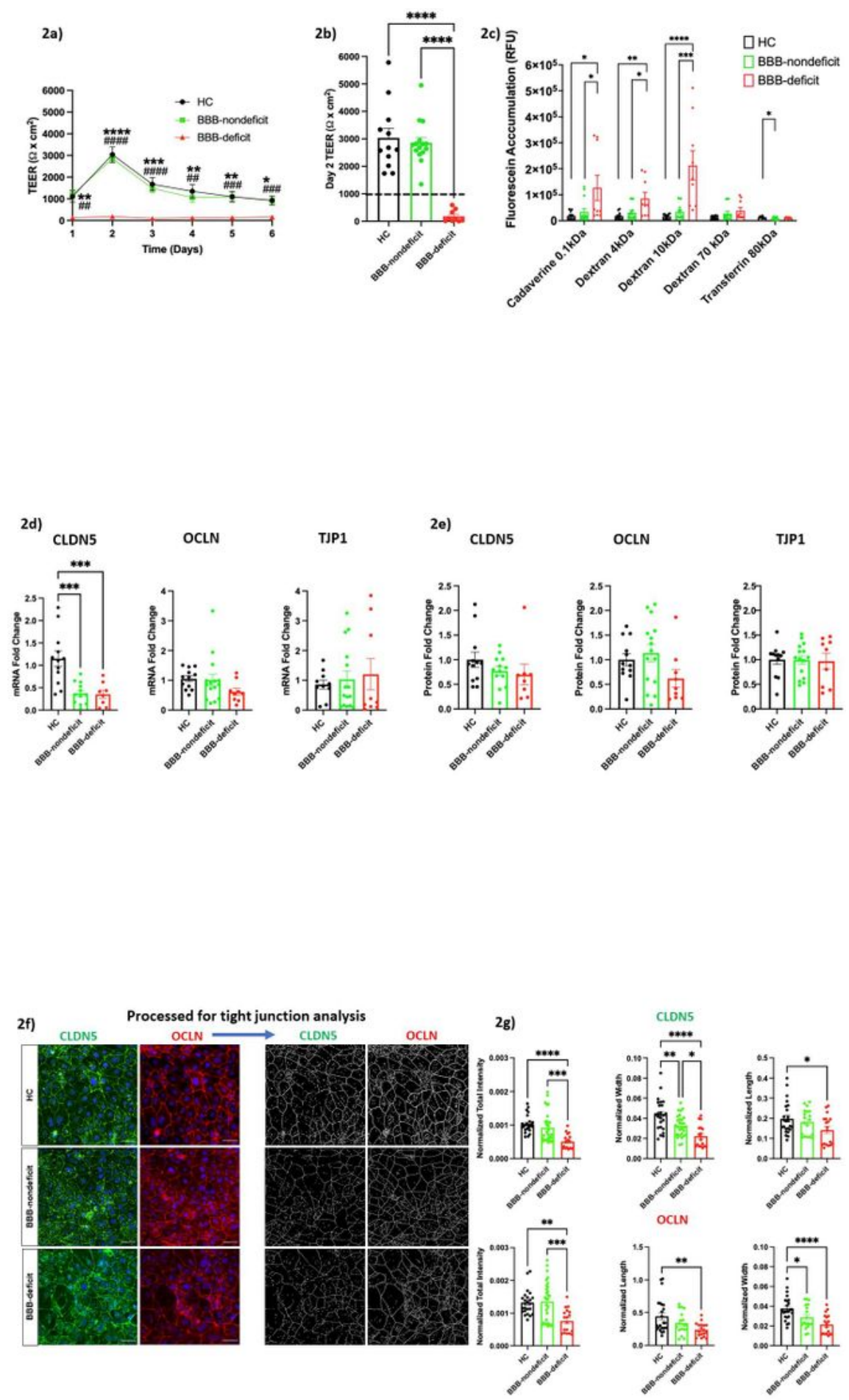

\section{Figure 2}

A psychosis subgroup shows extensive BBB leakage. a) Average TEER across 6 days with significant differences observed between HC $(n=4)$, BBB-nondeficit $(n=5)$ and BBB-deficit group $(n=3)$. BBB-nondeficit group consists of BD $(n=3)$ or $S Z(n=2)$ patients with TEER values greater than $1000 \Omega \times \mathrm{cm}^{2}$; BBB-deficit group consists of BD $(n=1)$ or $S Z(n=2)$ patients with TEER of less than $1000 \Omega \times \mathrm{cm}^{2}$. Significance was determined by Two-Way ANOVA and post-hoc Tukey multiple comparison test (** $\mathrm{p}=0.0097$ (day 1 ), 
${ }^{\star \star * \star \star} \mathrm{p}<0.0001$ (day 2), ${ }^{\star \star \star} \mathrm{p}=0.0009$ (day 3), ${ }^{\star \star} \mathrm{p}=0.0053$ (day 4), ${ }^{\star \star} \mathrm{p}=0.0068$ (day 5), ${ }^{\star} \mathrm{p}=0.0116$ (day 6) comparing BBB-deficit group to HC; and ${ }^{\# \#} \mathrm{p}=0.0012$ (day 1), ${ }^{\# \# \#} \mathrm{p}<0.0001$ (day 2), ${ }^{\# \# \#} \mathrm{p}<0.0001$ (day

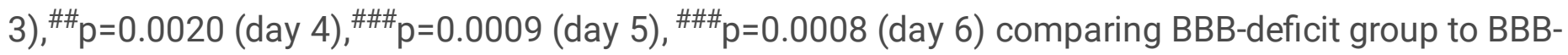
nondeficit group). b) Average peak (day 2) TEER values for HC, BBB-nondeficit, and BBB-deficit groups. Significance was determined by One-Way and post-hoc Tukey multiple comparison test $(* \star \star \star p<0.0001$ comparing BBB-deficit to HC or BBB-nondeficit groups). TEER values were collected using three biological replicates and shown as mean \pm s.e.m. C) Permeability of fluorescently labeled substrates for HC, BBBnondeficit, and BBB-deficit groups. Permeability values (RFU) were collected using three biological replicates and shown as mean \pm s.e.m. Significance was determined by One-Way and post-hoc Tukey multiple comparison test. Cadaverine $0.1 \mathrm{kDa}{ }^{*} \mathrm{p}=0.0107$ comparing BBB-deficit to $\mathrm{HC}$ and ${ }^{*} \mathrm{p}=0.0249$ comparing BBB-deficit to BBB-nondeficit; dextran 4kDa**p=0.0022 comparing BBB-deficit to HC and ${ }^{*} \mathrm{p}=0.0124$ comparing BBB-deficit to BBB-nondeficit; dextran 10kDa ${ }^{* \star \star} \mathrm{p}<0.0001$ comparing BBB-deficit to $\mathrm{HC}$ and ${ }^{* \star *} \mathrm{p}=0.0001$ comparing BBB-deficit to BBB-nondeficit; transferrin $80 \mathrm{kDa}{ }^{*} \mathrm{p}=0.0418$ comparing BBB-nondeficit to HC). d) Normalized mRNA levels for CLDN5, OCLN and TJP1 from HC, BBB-nondeficit, and BBB-deficit BMECs; mRNA expression for each gene was normalized to the housekeeping gene GAPDH. Each normalized value was then calibrated to the average mRNA expression from the HC group $(n=4)$ using the $\triangle \triangle C$ t method; RT-qPCR was performed in technical duplicates and each dot represents a biological replicate. Mean \pm s.e.m is shown for each group. Significance was determined by One-Way ANOVA and post-hoc Tukey multiple comparison test ( ${ }^{\star *} \mathrm{p}=0.0007$ comparing CLDN5 mRNA expression in BBB-deficit to $\mathrm{HC}$, ${ }^{\star \star *} \mathrm{p}=0.0003$ comparing CLDN5 mRNA expression in BBB-nondeficit to HC). 5 outliers were removed using the ROUT method ( $q=0.5$ ). e) Normalized levels of CLDN5, OCLN and TJP1 protein in HC, BBB-nondeficit, and BBB-deficit BMECs; each dot represents a biological replicate. Mean \pm s.e.m is shown for each group. Band intensities were quantified using Fiji by Image $\mathrm{J}$ and were normalized to GAPDH. Significance was determined by One-Way ANOVA and post-hoc Tukey multiple comparison test and no significant differences were observed between any of these groups. 3 outliers were removed. $f$ ) Representative images of immunostaining for CLDN5 (green), OCLN (red), and nuclei (blue) in BMECs from HC, BBB-nondeficit, and BBB-deficit groups; images were processed using Matlab, assessing for membrane and cytosolic fluorescence intensity; scale bar is $50 \mu \mathrm{m}$. g) Quantification of normalized total fluorescence intensity for width and length for CLDN5 and OCLN in HC, BBB-nondeficit, and BBB-deficit BMECs; 6 fields of view for each cell line were used for quantification. Raw intensity values were normalized to the total number of nuclei in each respective field of view. Each dot represents a field of view. Mean \pm s.e.m are shown for each group. Significance was determined by One-Way ANOVA and posthoc Tukey multiple comparison test. ${ }^{\star \star *} \mathrm{p}<0.0001$ comparing CLDN5 width in BBB-deficit to HC, ${ }^{\star \star} \mathrm{p}=$ 0.0046 comparing CLDN5 width in BBB-nondeficit to HC, $\mathrm{p}^{\star}=0.0251$ comparing BBB-deficit to BBBnondeficit; ${ }^{*} \mathrm{p}=0.0404$ comparing CLDN5 length intensity in BBB-deficit to $\mathrm{HC}$; ${ }^{\star \star \star *} \mathrm{p}<0.0001$ comparing CLDN5 total intensity in BBB-deficit to $\mathrm{HC}$, ${ }^{* \star} \mathrm{p}=0.0004$ comparing CLDN5 total intensity in BBB-deficit to BBB-nondeficit; $* \star \star \star p<0.0001$ comparing OCLN width in BBB-deficit to HC, ${ }^{*} \mathrm{p}=0.042$ comparing OCLN width in BBB-nondeficit to $\mathrm{HC},{ }^{* *} \mathrm{p}=0.0040$ comparing OCLN length in BBBO-deficit to $\mathrm{HC},{ }^{* \star} \mathrm{p}=0.0012$ comparing OCLN total intensity in BBB-deficit to $\mathrm{HC}$, ${ }^{\star \star \star} \mathrm{p}=0.0003$ comparing OCLN total intensity in BBBdeficit group to BBB-nondeficit. All reported p-values are two-sided. 
3a)

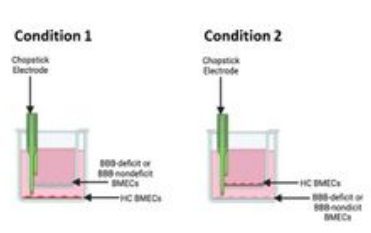

3b)

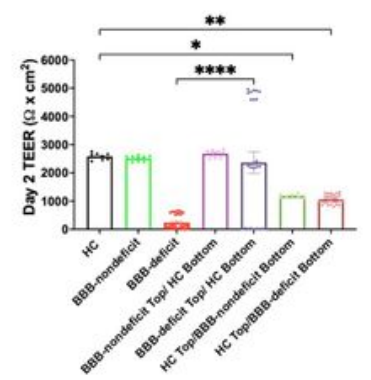

3c)

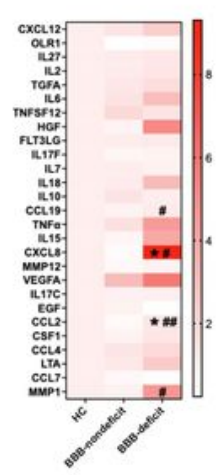

3d)

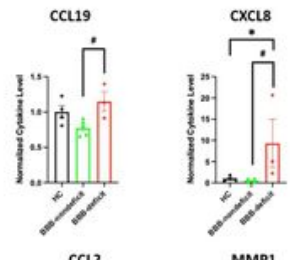

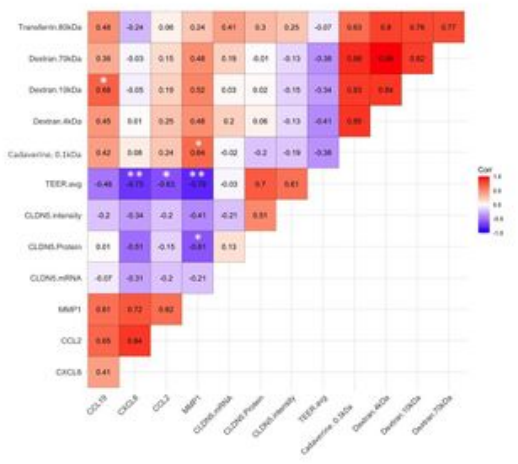

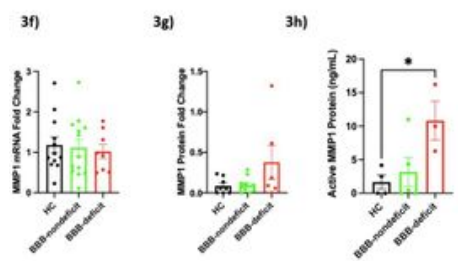

Figure 3

MMP1 secreted by SZ BMECs impairs BBB function. a) Schematic of cross-culture assays. In condition 1, BMECs from a $\mathrm{HC}$ individual was placed in the bottom chamber while BMECs from either a BBB-deficit or -nondeficit group was placed in the upper chamber. In condition 2, BMECs from a $\mathrm{HC}$ individual was placed in the top chamber while BMECs from either BBB-deficit or -nondeficit groups was placed in the bottom chamber. b) Peak (day 2) TEER values comparing HC subjects cultured alone to HC subjects co- 
cultured with either BBB-nondeficit or BBB-deficit subjects and comparing BBB-deficit subjects cultured alone to BBB-deficit subjects co-cultured with $\mathrm{HC}$ subjects. TEER values were collected using three biological replicates and shown as mean \pm s.e.m. Significance was determined by One-Way ANOVA and post-hoc Tukey multiple comparison test $\left({ }^{*} \mathrm{p}=0.0466\right.$ comparing HC Top/BBB-nondeficit Bottom to HC cultured alone, ${ }^{*} \mathrm{p}=0.0019$ comparing HC Top/BBB-deficit Bottom to HC cultured alone, ${ }^{* \star \star *} \mathrm{p}<0.0001$ comparing BBB-deficit Top/ HC Bottom to BBB-deficit cultured alone. c) Heatmap depicting normalized proteomic marker levels detected in conditioned media collected from $\mathrm{HC}(n=4)$, BBB-nondeficit $(n=5)$, and BBB-deficit ( $n=3$ ) groups during peak TEER (day 2) values. d) Normalized levels of CCL19, CXCL8, CCL2, and MMP1 in HC, BBB-nondeficit, and BBB-deficit BMECs. Raw concentration values were normalized to the average concentration of individuals in the $\mathrm{HC}$ group. Each dot represents the normalized cytokine level for each subject. Mean \pm s.e.m is shown for each group. Significance was determined using a general linear hypothesis test ${ }^{*} p=0.0143$ comparing CCL19 in BBB-deficit to BBBnondeficit, ${ }^{*} \mathrm{p}=0.0453$ comparing CXCL8 in BBB-deficit to $\mathrm{HC}$, ${ }^{*} \mathrm{p}=0.0289$ comparing CXCL8 in BBB-deficit to BBB-nondeficit, ${ }^{*} \mathrm{p}=0.0280$ comparing CCL2 in BBB-nondeficit to $\mathrm{HC},{ }^{\# \#} \mathrm{p}=0.00369$ comparing CCL2 in BBB-deficit to BBB-nondeficit, ${ }^{*} \mathrm{p}=0.0345$ comparing MMP1 in BBB-deficit to BBB-nondeficit, and $\mathrm{p}=0.0516$ comparing MMP1 in BBB-deficit to HC. e) Correlation matrix between peak TEER values, CLDN5 mRNA and protein levels, fluorescein accumulations from permeability assay, and the concentrations of markers from proteomic analysis. Correlations were performed using Spearman correlations (CCL19Dextran 10kDa $r=0.678,{ }^{*}=0.0118$; CXCL8-TEER $r=-0.734$, ** $p=0.0091$; CCL2-TEER $r=-0.69,{ }^{*} p=0.0324$, MMP1-TEER $r=-0.790,{ }^{*} \mathrm{p}=0.0036$; MMP1-CLDN5 protein level $\mathrm{r}=-0.608,{ }^{*} \mathrm{p}=0.040$; MMP1-Cadaverine $0.1 \mathrm{kDa} r=0.643,{ }^{\star} \mathrm{p}=0.0280$ ). (f-h) Quantification of MMP1 mRNA, protein, and proteolytic activity in HC, BBB-nondeficit, and BBB-deficit groups. Mean \pm s.e.m is shown for each group. Significance was determined by One-Way ANOVA and post-hoc Tukey multiple comparison test ( $\left.{ }^{*} p=0.419\right)$. RT-qPCR was conducted in three technical and biological replicates for each subject. MMP1 mRNA expression was normalized to the housekeeping gene GAPDH. Each normalized value was then calibrated to the average MMP1 mRNA expression from the $\mathrm{HC}$ group $(\mathrm{n}=4)$ using the $\triangle \triangle \mathrm{Ct}$ method. Western blots were performed in two biological replicates for each subject and band intensities were quantified using Fiji by Image $J$ and band intensities were normalized to GAPDH. MMP1 protein activity assay (human Active MMP1 Flurokine $\mathrm{E}$ kit) was performed in technical triplicates using conditioned medium from HC, BBB-nondeficit, and BBB-deficit BMECs. All reported p-values are two-sided. 
4a)
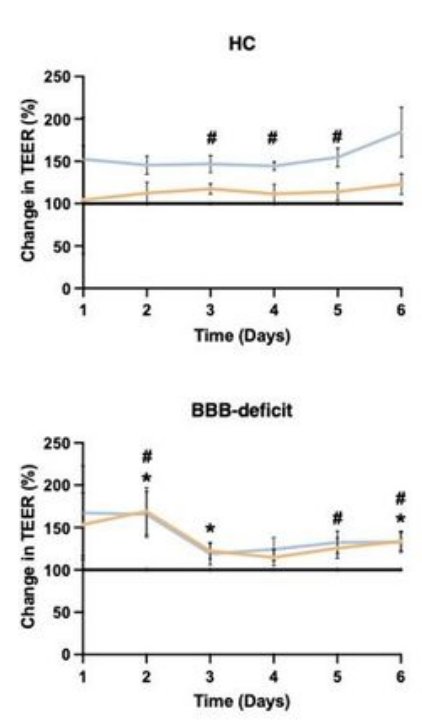

4c)

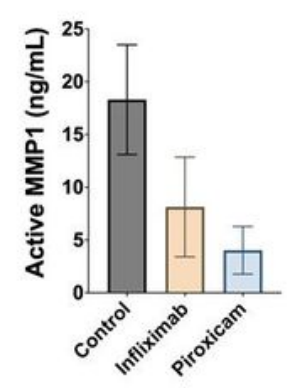

4b)

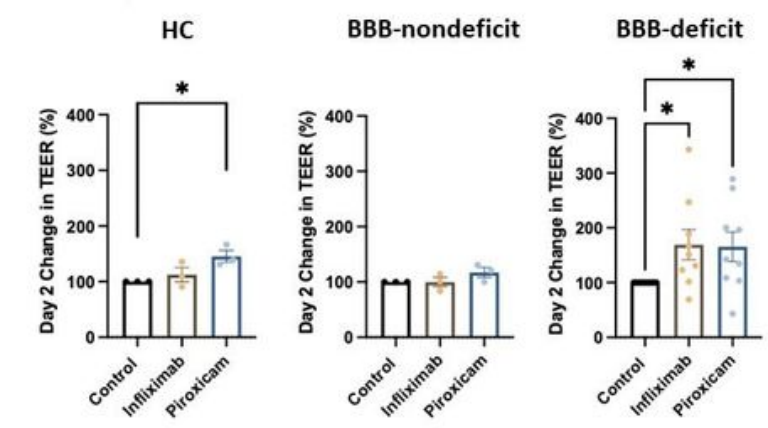

\section{- Control \\ - Infliximab \\ - Piroxicam}

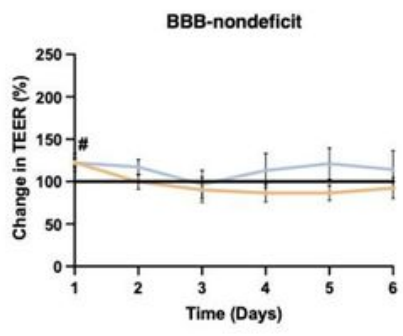

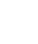

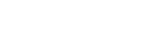

4d)

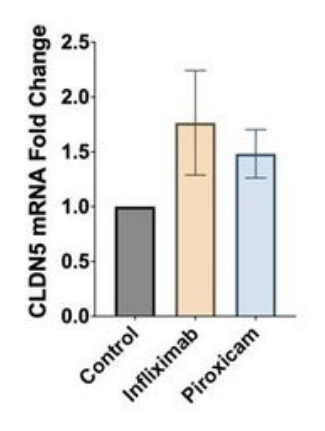

\section{Figure 4}

Inhibition of MMP1 and TNFa restores BBB function. a) Percent change in TEER across six days in HC $(n=1)$, BBB-nondeficit $(n=1)$, and BBB-deficit $(n=3)$ groups treated with pharmacologic MMP1 inhibitor (Piroxicam) or biologic TNFa inhibitor (Infliximab). BMECs were exposed to Infliximab $(300 \mathrm{ng} / \mathrm{mL}$ ) or Piroxicam(10uM) for 24 hours during subculturing (day 6). Percent change was calculated based on the average TEER value in infliximab or piroxicam condition from each day and then normalized to the 
average TEER value in the control condition. Significance determined by Two-Way ANOVA and Uncorrected Fisher's LSD multiple comparison test ( $\mathrm{HC}$ group: day $3{ }^{*} \mathrm{p}=0.0437$ comparing piroxicam to control, day $4{ }^{\#} \mathrm{p}=0.0112$ comparing piroxicam to control, day $5{ }^{{ }^{*}} \mathrm{p}=0.0395$ comparing piroxicam to control; BBB-nondeficit group: day $1{ }^{*} \mathrm{p}=0.0489$ comparing piroxicam to control; BBB-deficit group: day 2 ${ }^{*} \mathrm{p}=0.0371$ comparing infliximab to control and ${ }^{*} \mathrm{p}=0.0413$ comparing piroxicam to control, day 3 ${ }^{*} \mathrm{p}=0.0494$ comparing infliximab to control, day $5{ }^{*} \mathrm{p}=0.0441$ comparing piroxicam to control, day 6 ${ }^{*} \mathrm{p}=0.0153$ comparing infliximab to control and ${ }^{\#} \mathrm{p}=0.0309$ comparing piroxicam to control). b) Peak (day 2) TEER values of BMECs from HC, BBB-nondeficit, and BBB-deficit groups with infliximab or piroxicam treatment. Significance was determined by One-Way ANOVA and Uncorrected Fisher's LSD multiple comparison test (HC group: ${ }^{*} \mathrm{p}=0.0161$ comparing piroxicam to control; BBB-deficit group: ${ }^{*} \mathrm{p}=0.0385$ comparing infliximab to control and ${ }^{*} \mathrm{p}=0.0487$ comparing piroxicam to control). TEER values were collected using three biological replicates and shown as mean \pm s.e.m. c) Quantification of MMP1 protein activity in conditioned medium from BMECs of BBB-deficit subjects with infliximab or piroxicam treatment. MMP1 protein activity assay was performed in technical duplicates. d) Quantification of CLDN5 mRNA expression in BMECs from BBB-deficit subjects treated with infliximab or piroxicam; RTqPCR was conducted in three technical and biological replicates for each line. Mean \pm s.e.m is shown for each group. By using One-Way ANOVA and post-hoc Tukey multiple comparison test, no significances were observed in MMP1 activity or CLDN5 mRNA levels. All reported p-values are two-sided.

\section{Supplementary Files}

This is a list of supplementary files associated with this preprint. Click to download.

- SuppFigureLegends.docx

- SuppFigsTables.pptx 